\title{
SOBRE EL DISCURSO INSTRUCCIONAL
}

\author{
Mario Díaz V.
}

\section{Introducción}

Este artículo se relaciona con el discurso instruccional. Aquí intentaremos explicar cómo la distribución del poder y los principios de control regulan la producción, reproducción y cambios del discurso instruccional a través de la compleja red de relaciones que dichos principios establecen. Intentaremos explicar también cómo estos principios crean una ubicación desigual de los sujetos en los discursos instruccionales de la escuela, una ubicación desigual de sus competencias culturales y, por lo tanto, su ubicación desigual en el campo de producción y en el campo de control simbólico.

El análisis del discurso instruccional tradicionalmente ha sido confinado a sus rasgos superficiales. En general, éste ha sido considerado como una selección de significados con base en criterios lógicos, racionales e intrínsecos a las disciplinas y relevantes para el desarrollo de la mente (Hirst, 1965, 1969; Peters, 1965; Hirst y Peters, 1970); o como los bienes culturales (capital cultural) transmitidos por la escuela (Bourdieu, 1977); ó como la organización escolar del conocimiento socialmente prescrito (aquel que constituye la cultura dominante) (Green, 1971). El confinamiento del estudio del discurso instruccional a sus rasgos superficiales -lo que Young (1975) Ilama curriculum como "hecho" no ha permitido comprender la relación entre los principios y reglas (exclusión, inclusión, apropiación, distribución) que regulan las relaciones dentro de y entre los discursos instruccionales tanto en su producción como en su reproducción, y las formas de conciencia con las cuales dichos principios y reglas se relacionan (Bernstein, 1981).

Una forma de exploración diferente debe mostrar como intrínseco al discurso instruccional y a su producción/reproducción existe todo un conjunto de principios organizativos que ubican a los sujetos con respecto a los rasgos internos y relacionantes de competencias especializadas. Esta gramática semiótica ubicadora específica regula la realización desigual de aquellas competencias en la sociedad.

En una forma muy descriptiva podemos decir que el discurso instruccional regula las reglas que constituyen la variedad legítima, los rasgos internos y las relaciones de competencias especializadas (Bernstein, Seminarios) ${ }^{253}$. Aquí nos referiremos al discurso instruccional oficial, es decir, al discurso instruccional distribuido dentro de la educación formal. Consideramos el discurso instruccional oficial como algo separado del discurso instruccional que regula el aprendizaje en otros contextos sociales tales como la familia, la comunidad. Llamamos a este último discurso, discurso instruccional local.

\footnotetext{
${ }^{253}$ La noción de discurso Instruccional tiene sus fuentes en el análisis de Parsons sobre las actividades instrumentales y los roles $(1951,1955,1959)$ y en los desarrollos de Bernstein desde una perspectiva diferente. Bernstein usa por primera vez el término Instrumental en su artículo "Sources of consensus and disaffection in Education" (1966) en el cual distingue la cultura instrumental de la cultura expresiva. Aquí la cultura Instrumental se refiere a "los hecho., procedimientos y juicios envueltos en la adquisición de habilidades específicas". El trabajo sociolingüístico de Bernstein, le permite distinguir cuatro contextos de socialización entre los cuales el 'contexto instruccional' se refiere a aquel contexto en el cual "el niño aprende acerca de la naturaleza objetiva de las cosas y personas y aprende habilidades". Sin embargo, es en Pedro (1981) donde los comienzos de un modelo de análisis del discurso Instruccional es tomado de Bernstein y donde éste último define el discurso instruccional en términos de "los principios del discurso específico susceptibles de ser transmitidos y adquiridos".
} 


\section{Características generales del discurso instruccional}

El discurso instruccional presupone una previa división social de los transmisores, adquirientes, discursos y sitios de reproducción, es decir, presupone un principio fundamental de clasificación. Sin embargo, el discurso instruccional puede iniciar bajo ciertas condiciones un cambio en el principio de clasificación. Desde este punto de vista, el discurso instruccional presupone relaciones de poder y al mismo tiempo legitima y reproduce estas relaciones. Por ejemplo, cuando las materias escolares están rígidamente clasificadas, en el sentido que constituyen discursos distintos y jerarquizados, entonces, cualquier discurso instruccional que esté relacionado con el cambio de las relaciones entre discursos, o entre maestros y alumnos, se encontrará a sí mismo en conflicto con la distribución del poder que se constituye en las relaciones entre los discursos, maestros, alumnos y sitios existentes ${ }^{254}$.

Además, el discurso instruccional presupone el proceso de recontextualización mediante el cual los discursos han sido históricamente removidos del campo de su producción, transformados y transferidos al campo educativo o de reproducción ${ }^{255}$. Este proceso de recontextualización es relativamente complejo e implica un campo de recontextualzación oficial y un campo de recontextualización pedagógica (Bernstein y Díaz, 1985). Desde esta perspectiva, el discurso instruccional es aquel discurso producido en las tensiones entre estos dos campos. Mientras que el campo de recontextualización oficial determina los criterios formales y las normas de validez que se emplean como leyes para la legitimación de un discurso instruccional, el campo de recontextualización pedagógica es un espacio para la reestructuración, reenfoque y cambio de los discursos instruccionales. La autonomía relativa de este último campo permite un mayor control sobre la circulación de teorías y prácticas del contexto primario de producción del discurso al contexto de su reproducción (Véase, Bernstein y Díaz, 1985).

El discurso instruccional también presupone aquellas relaciones sociales por medio de las cuales se realizan las prácticas instruccionales.

El discurso instruccional, pues, debe considerarse como un dispositivo ubicado y ubicador. Usando los términos de Bernstein, podemos considerar que el sujeto es ubicado diferencialmente por los códigos intrínsecos a la selección, transmisión y evaluación del discurso instruccional. Si, por una parte, el principio de clasificación al actuar sobre las relaciones entre los discursos instruccionales crea relaciones de posición/oposición específicas de los sujetos dentro de éstos (identidades específicas, voces específicas, poder discursivo), por la otra, el principio de enmarcación, como veremos más adelante, regula la lógica de la transmisión/adquisición del discurso instruccional ${ }^{256}$. A partir de esto se deduce que el código instruccional es un regulador de las relaciones entre discursos

\footnotetext{
${ }^{254}$ Goodson (1981) muestra, por ejemplo, cómo la geografía progresó desde una materia escolar de bajo status en el siglo XIX hasta una disciplina académica con un status y base universitaria. Goodson considera que la lucha por el status es sobre todo "una batalla por los recursos materiales, perspectivas y carrera de cada profesor de una materia, o de una comunidad de materias".

${ }^{255}$ Aquí nos referimos al discurso Instruccional como el compendio de una compleja red de relaciones sociales. No es, pues, nuestra intención mistificar con este discurso la tradicional y casi monolítica explicación de la unilateral relación entre materias y disciplinas. Esta diferencia, ciertamente, no es ni estable, ni constante, ni absoluta. (Véase Goodson, 1981. Tamblén, véase la nota de Foucault sobre el "comentario", en Foucault, 1970.

${ }^{256}$ Esta distinción apunta a una diferencia fundamental entre la ubicación del discurso Instruccional y la ubicación dentro del discurso instruccional. La ubicación del discurso instruccional envuelve un principio de clasificación y un principio de recontextualización. La ubicación dentro del discurso Instruccional básicamente se refiere a la realización de las competencias de sujetos culturales específicos en dicho proceso de ubicación.
} 
instruccionales pero también un regulador de las relaciones dentro de un discurso instruccional dado. Lo que cuenta como un discurso instruccional depende del principio de clasificación y lo que cuenta como transmisión legítima de un discurso instruccional dado depende de los valores de la clasificación y la enmarcación del contexto instruccional.

Invisiblemente presentes en un discurso instruccional se encuentran dos grupos de teorías que pueden o no estar relacionadas: un grupo de teorías regula "el qué" de la transmisión y otro regula "el cómo".

El Qué. Aquí debemos distinguir dos procesos no necesariamente relacionados. Un proceso se refiere a la selección de los discursos que constituyen un conjunto de discursos instruccionales dados; aquí tenemos un principio clasificatorio fundamental el cual especializa el conjunto. Un segundo principio clasificatorio regula el ordenamiento horizontal y vertical del conjunto. Detrás de estos principios clasificatorios existe un proceso de recontextualización. Desde esta perspectiva un currículum es la realización superficial de este complejo proceso de regulación.

El principio de clasificación es el principio que regula la formación, ordenamiento, distribución y contextos de un conjunto dado de discursos instruccionales al fijar sus límites, redefinir sus fronteras y dar origen a la política de constitución de discursos instruccionales. Podemos distinguir la siguiente clasificación a lo largo de una dimensión fuerte:

a) La fuerza de la clasificación del conjunto de discursos y sus relaciones con la clasificación interna de éste. (Un conjunto puede consistir de un solo discurso en cuyo caso éste representaría la clasificación más fuerte).

b) El número de lo que cuenta como discursos diferentes en el conjunto. Los conjuntos varían de acuerdo con las reglas de exclusión y de inclusión.

c) La estabilidad del conjunto en el tiempo.

d) El enfoque del conjunto, manual/mental, "culto" "especialista" "pastoral", "vocacional", etc. El enfoque puede establecer una dicotomía entre orientaciones hacia los significados elaboradas y restringidas.

e) El número de conjuntos diferentes, las relaciones entre éstos y el grado en el cual tales conjuntos se especializan para grupos de adquirientes.

El Cómo. Aquí nos referimos a las teorías de transmisión o instrucción y a las teorías del aprendizaje, las cuales implican un proceso de recontextualización y, por lo tanto, una reubicación ideológica de diversas teorías psicológicas.

Las teorías acerca del "qué" subyacen al principio intrínseco de clasificación de los discursos instruccionales (formas de conocimiento, campos de conocimiento, disciplinas en términos de Foucault). Las teorías del "como" subyacen al principio que regula la lógica de la transmisión/adquisición de los discursos instruccionales, (valores de la clasificación y de la enmarcación). Las teorías del "qué" y del "como" pueden encontrarse en relaciones de dominación/subordinación entre sí. Es posible, por ejemplo que una teoría de la adquisición (del aprendizaje) domine la teoría de la transmisión y ocasione un cambio tanto en el conjunto de discursos como en su ordenamiento interno. Igualmente, es posible que el "qué" pueda dominar la selección del "cómo". A este respecto, Bernstein 
ha argumentado que si vamos a discutir el "qué" debemos también discutir el "cómo" (pedagogía y evaluación). El "qué" y el "cómo" forman un conjunto y deben tratarse como un todo.

La lógica social de la producción/reproducción del discurso instruccional. Las bases de la recontextualización

Hemos dicho en otra parte que la producción/reproducción del discurso instruccional conlleva agentes, agencias, posiciones y prácticas dentro del campo de recontextualización, el cual — de acuerdo con Bernstein- está regulado por la división del trabajo y sus relaciones sociales intrínsecas.

El principio que regula las relaciones entre discursos instruccionales, es decir, el principio que regula la división social del trabajo del discurso mstruccional es el principio de clasificación. Este- principio asegura la reproducción de las relaciones de poder mediante la demarcación, selección, exclusión y jerarquización, ordenamiento y distribución de los discursos que deben reproducirse. Además, esta regulación también conlleva un principio de recontextualización por el cual los discursos y prácticas son desubicados en el campo educativo de reproducción.

Los principios de clasificación y de recontextualización crean para el conocimiento una nueva organización estructural y jerárquica un nuevo orden lógico (secuencia) que se define en correspondencia con el orden y progreso de la adquisición/transmisión. Encontramos aquí una distinción crucial regulada por una clasificación rígida entre lo que podría denominarse "la lógica social de la producción del discurso" y "la lógica social de su reproducción.

Por "lógica social de la producción del discurso" entendemos el proceso por el cual agentes, posiciones, discursos y prácticas constituyen un campo especializado dedicado a la producción y elaboración de lo que se denomina como nuevo conocimiento. Generalmente existe una clasificación rígida entre aquellos que producen y aquellos que reproducen. Esto significa que los maestros son especialistas en la reproducción del conocimiento más que en su producción. Los textos escolares raras veces son escritos por aquellos que producen conocimientos. Estos son escritos por aquellos que reproducen o que recontextualizan el conocimiento.

La "lógica social de reproducción del discurso" está relacionada con la regulación de las prácticas de transmisión/adquisición. Cuando las dos lógicas, de producción y de reproducción, están fuertemente clasificadas, debe desarrollarse un campo de recontextualización con sus propias posiciones, funciones, y discursos internos. Las prácticas de des-ubicación y re-ubicación de los discursos que constituyen la actividad esencial de este campo dependen de su relativa autonomía de las propias agencias y políticas del estado, del enfoque de estas políticas, y del grado en el cual los agentes externos (maestros y comunidad) están subordinados, opuestos, o articulados a las prácticas de este campo. En otras palabras, el continuo proceso de recontextualización depende de la lógica de las posiciones y oposiciones internas del campo, pero también de la relativa autonomía de dichas posiciones y oposiciones del estado. (Es claro que no podemos excluir como una fuerza determinante la dinámica tanto del campo de producción como del campo de control simbólico).

Desde esta perspectiva es posible distinguir dos campos de recontextualización interrelacionados: el campo de recontextualización oficial y el campo de 
recontextualización pedagógica ${ }^{257}$. El campo de recontextualización oficial es básicamente dependiente del sistema jurídico-político y administrativo del estado. Sus agencias, generalmente, están localizadas en, o reguladas por, el Ministerio de Educación - $u$ otros Ministerios- y se relaci\&nan con la creación de regulaciones, controles y políticas de circulación de los discursos, teorías y prácticas. El campo de recontextualización pedagógica puede considerarse como un conjunto estable y relativamente autónomo de agentes/agencias que regulan la circulación de teorías/discursos/prácticas desde el contexto primario de eu producción hasta el contexto de reproducción pedagógica.

Las relaciones dentro de, y entre los campos de recontextualización oficial y de recontextualización pedagógica tienen implicaciones para la recontextualización de discursos/prácticas susceptibles de ser reproducidos. Es dentro de este conjunto de relaciones complejas que se constituye lo que podríamos llamar el discurso pedagógico oficial.

Ahora podemos decir que el discurso jnstruccional oficial es un dispositivo institucionalizado y legítimo, recontextualizado y recontextualizante, cuyos principios y reglas regulan $y$, por lo tanto, fijan los límites de la realización legítima de las competencias de los sujetos culturales específicos en la escuela. Las modalidades de ubicación en el discurso instruccional oficial -que dependen de las modalidades de ubicación del discurso instruccional en la escuela- están reguladas por las prácticas instruccionales oficiales seleccionadas y hechas públicas o especificadas en las regulaciones, guías y toda clase de textos pedagógicos producidos tanto por las agencias pedagógicas del estado como por las agencias del campo de recontextualización pedagógica ${ }^{258}$.

Intrínsecas a la división social del trabajo de producción del discurso instruccional existen relaciones de clasificación verticales y horizontales. La clasificación vertical regula la estratificación de los discursos (lo "mental" se considera como más importante que lo "manual"), y la clasificación horizontal regula la relación entre los discursos en términos de su grado de especialización. Esta clasificación que, generalmente, ocurre a un nivel epistémico puede también conducir a pugnas por el control intelectual sobre determinadas áreas discursivas.

Además, la clasificación de los discursos y su agrupamiento en el campo de producción del discurso puede tener solamente una relación aproximada con los discursos y su agrupamiento en el contexto de reproducción. En el proceso de recontextualización los

\footnotetext{
${ }^{257}$ El campo de recontextualización es, en esencia, pedagógico en su función acordada de regulación de la circulación de los textos. En las sociedades contemporáneas el creciente mercado de la cultura ha conducido a un incremento en las agencias de recontextualización y a oposiciones entre ellas. Pensemos, por ejemplo, en las contradicciones generadas entre el "aparato cultural" y la llamada "industria de la conciencia" (Gouldner, 1976). La mercantilización de la cultura ha producido un voluminoso mercado de textos recontextualizados (revistas periódicas, libros de bolsillo, magazines, textos escolares, revisión de libros, colecciones selectas, etc.). El incremento en la pedagogización de los textos ha creado nuevas modalidades de consumo cultural -utilizando el término de Bourdieu-. Esta pedagogización mantiene, reproduce y legitima la distribución desigual del acceso a los recursos culturales o simbólicos

${ }^{258}$ En países donde existe una autoridad centralizada y una dirección política del estado sobre la selección, transmisión y evaluación del discurso instruccional, las agencies pedagógicas del estado organizadas sobre principios burocráticos específico. constituyen una poderosa maquinaria para la organización y control (directo o indirecto) de los niveles, posiciones, sitios, discursos y prácticas del contexto de reproducción. Medio, tales como regulaciones, normas, planes, proyectos, decretos, recomendaciones, y otra clase de textos oficial.., provisto, por las agencias pedagógicas y legitimados en virtud de regulaciones oficiales, constituyen el dominio de lo que puede denominarse como discurso pedagógico oficial.
} 
significados - dice Bernstein - son reunidos y, por supuesto, separados en una forma particular (Bernstein, 1977). Así, pues, los discursos instruccionales, generados mediante formas distintas de relaciones sociales, son a su vez generadores de formas distintas de relación social en el contexto de reproducción.

Históricamente ha existido una separación entre "mental" y "manual" dentro del discurso instruccional, creándose así una clasificación rígida entre las prácticas intelectuales (elaboradas) y las prácticas manuales (restringidas) ${ }^{259}$. Esto a su vez ha mantenido y reproducido una clasificación rígida entre el campo de producción de recursos físicos y el campo de producción de recursos discursivos. También ha provocado dentro del campo de producción de recursos discursivos una clasificación fuerte entre los agentes y prácticas intelectuales y los agentes y prácticas manuales.

Las prácticas intelectuales han sido, en general, constituidas por el discurso "académico" adquirido directa o indirectamente a través de la escolaridad ${ }^{260}$. Las prácticas manuales fueron adquiridas fuera de la escuela, bien en la familia, bien en los talleres. Históricamente, la práctica pedagógica estuvo esencialmente -y únicamenterelacionada con la práctica intelectual. Sin embargo, las escuelas modernas proporcionan prácticas vocacionales como, por ejemplo, manualidades y destrezas, pero estas escuelas a menudo distribuyen tales prácticas de acuerdo con la clase social y el género de los alumnos. Esto plantea el interrogante de qué manualidades, qué destrezas, cuándo, para quién, y cómo, y también el interrogante de sus principios de recontextualización. El contexto primario para estas prácticas es, por supuesto, no el campo intelectual sino el campo de producción.

Las prácticas manuales escolares pueden tener tan poca relación con las prácticas reales del campo de producción como las prácticas intelectuales de la escuela con las prácticas reales del campo intelectual. Aquí, tal vez podemos percibir cómo las prácticas ideológicas de las agencias de recontextualización crean una relación imaginaria entre el campo primario de producción y el campo educativo de reproducción.

Así, si miramos el proceso histórico de reestructuración del campo económico (campo de producción) podremos observar la recontextualización de' las prácticas manuales y su reubicación en el paradigma instruccional en la forma de discurso vocacional (con sus realizaciones técnicas). El discurso vocacional se convierte en un requisito para la reproducción de habilidades específicas (su concepto central), pero también para la reproducción de formas de conciencia. En otras palabras, incluye el concepto de realización de habilidades de trabajo (Vaizey, 1963) en el concepto de habilidades sociales y para la vida, lo cual, a su vez, presupone el cambio de la ubicación de los agentes en el mercado de trabajo. Desde esta perspectiva, podemos ver que el discurso vocacional —como una realización del discurso instruccional- se ha convertido en un portador del discurso regulativo.

\footnotetext{
${ }^{259}$ La distinción entre trabajo mental y trabajo manual no se deriva de rasgos Intrínsecos a estas prácticas sino de la división social del trabajo y de las relaciones sociales de producción/reproducción. Desde esta perspectiva, el mantenimiento de la clasificación rígida entre trabajo mental y trabajo manual es fundamental para la reproducción de las relaciones de clase. (Bernstein, 1977, 1981); Poulantzas (1976); Wright (1978).

${ }^{260}$ El sistema más antiguo de discurso instruccional que conocemos —el discurso académico- fue inicialmente constituido en la Edad Media. se establecieron relaciones jerárquicas entre discursos especializados (las siete artes), las cuales no gozaban del mismo status; estas fueron divididas en dos grupos cuyo significado educativo fue diferente: el trivium que consistía en la enseñanza de aquellas disciplinas cuyo objeto lo constituían las diversas manifestaciones de la naturaleza humana: la gramática, la dialéctica y la retórica. El quadrivium agrupaba la totalidad de las disciplinas que se relacionaban con los objetos o las cosas: la aritmética, la geometría, la música y la astronomía (Durkheim, 1977). 
Es importante notar cómo las escuelas modernas proporcionan, además de las prácticas vocacionales, un conjunto diverso de discursos y prácticas dentro del discurso instruccional oficial. Algunos de estos discursos/prácticas tienen una función regulativa explícita como, por ejemplo, salud, servicio social o comunitario, economía del hogar, mientras que otros tienen una función regulativa implícita (programas de entrenamiento de jóvenes). Otros discursos/prácticas envuelven su función regulativa en una función más expresiva, o aún, en una función recreativa, (pensemos en los programas de recreación modernos) $)^{261}$.

Las diferentes realizaciones del discurso instruccional —académico, vocacional, expresivo y práctico no-vocacional (manualidades) tienen diférentes grados de relevancia para los campos de producción y de control simbólico ${ }^{262}$.

Fundamentalmente, el discurso vocacional recontextualiza las prácticas (habilidades) envueltas en la producción material. El discurso vocacional se ha convertido en una constante metáfora para el campo de producción; el discurso académico básicamente recontextualiza diferentes dominios del conocimiento, las tan llamadas ciencias/humanidades que tienen una relevancia directa/indirecta tanto para el campo de producción como para el campo de control simbólico. Históricamente, el discurso académico ha mido asociado con el concepto de educación liberal. Su clasificación fuerte con respecto al discurso vocacional se manifiesta en el hecho de que el discurso académico nunca ha estado comprometido con ninguna tarea vocacional (Durkheim, 1977; Hirst, 1974). El discurso expresivo podría considerarse como un conjunto de prácticas recontextualizadas que pueden agruparse en lo que arbitrariamente llamaremos "el campo expresivo" o "las artes expresivas" (visuales, acústicas, gráficas, cinéticas). Al recontextualizar las reglas técnicas o los principios teóricos que las caracterizan, la escuela no solamente reproduce el carácter legítimo de estas artes sino que en forma selectiva intenta reproducir los modelos culturales dominantes del campo cultural de la sociedad $^{263}$. El discurso expresivo sería, entonces, una recontextualización del campo cultural de la sociedad. En la medida en que la esfera de legitimidad de este campo se ha expandido ("a través de la legitimación legítima o a través de la competencia social por la legitimación", Bourdieu, 1968) asimismo se ha expandido el discurso expresivo de la

\footnotetext{
${ }^{261}$ Las propuestas modernas para la organización del discurso vocacional han Introducido un área Instruccional relacionada con ".el trabajo, el goce —o recreación-y los estilos de vida". Esta área incluye prácticas para el desarrollo y cambio de "las relaciones humanas, el tiempo libre, y también intereses tales como la habilidad para conducir un vehículo, elaborar un presupuesto, organizar las compras, etc. Esta nueva modalidad de discurso/práctica se ha constituido alrededor de la noción de "preparación para la vida". (Véase Skilbeck, 1980).

262 La esfera de legitimidad del discurso Instruccional depende de las transformaciones de la sociedad y de las complejas relaciones que tales transformaciones producen tanto en el campo de producción como en los campos de control simbólico y de recontextualización. Así, cuando una sociedad sufre cambios tecnológico. rápidos, puede. estrecharas la relación entre, el campo d recontextualiszación y el campo de producción mediante la expansión de los discursos centrados alrededor de habilidades destrezas y vocaciones. Sin embargo, bajo períodos de fuerte desempleo el discurso instrucional aumenta su función regulativa, esto es, deviene un dispositivo de control social. La ubicación de los sujetos en el conocimiento se vuelve menos Importante que la ubicación de los sujetos en la sociedad.

${ }^{263}$ A este respecto, Bourdieu declara lo siguiente: "La escuela tiene la función de perpetuar y transmitir el capital de los signos culturales consagrados, esto se, la cultura manejada por los creadores intelectuales del pasado y organizar sus prácticas en correspondencia con los modelos de esta cultura (...) Además, la escuela está obligada a establecer y definir sistemáticamente la esfera de la cultura ortodoxa y la esfera de la cultura heterodoxa. Simultáneamente, la escuela defiende la cultura consagrada contra el continuo reto provocado por la existencia de nuevos creadores quienes originan en el público nuevas demandas y dudas rebeldes" (Bourdieu, 1968).
} 
escuela. Sin embargo, las jerarquías se mantienen. Comparemos la diferencia entre la múmica clásica, el jazz, y la música folclórica (Vulliamy, 1976) ${ }^{264}$.

Algunas realizaciones del discurso expresivo se derivaron inicialmente de una función regulativa. Así, por ejemplo, los estudios de danza tuvieron una base psicológica/terapéutica en los comienzos del siglo XX en Inglaterra en la forma de danza conocida como "danza educativa moderna". Sin embargo, entre los años sesenta y setenta se fue legitimando la función estética de esta danza (Adshead, 1981).

La categoría que arbitrariamente hemos denominado discurso práctico no-vocacional sería una recontextualización de las técnicas y prácticas adoptadas en la configuración y manipulación de materiales. En otros términos, esta categoría se refiere a la regulación de la instrucción manual en el uso de herramientas y en prácticas como modelaje, dibujo, carpintería, etc. Esta área particular de discurso/práctica ha sido históricamente excluida de la "educación estética general" y se ha convertido en una introducción elemental a la educación vocacional actuando como un dispositivo de selección social y como un medio de control simbólico.

Como podemos ver, el discurso instruccional ha incrementado el rango de sus discursos y, por lo tanto de sus prácticas que regula y reproduce. Tal vez, no es mucho decir que pocas áreas de la vida social han quedado sin pedagogizar. También, podemos ver que con la creciente complejidad y expansión del discurso instruccional ha habido una extensión del papel de este discurso en la transmisión/adquisición del orden regulativo. Aquí podemos argumentar la penetración del discurso instruccional por el discurso regulativo, o la reproducción del orden regulativo a través del discurso instruccional.

La lógica social de la reproducción del discurso instruccional. Las bases regulativas de las prácticas instruccionales

La división social del trabajo y las relaciones sociales creadas para la transmisión/reproducción del discurso instruccional se establece mediante los principios de clasificación y enmarcación. Estos principios regulan la estructura (organización), interacciones y contextos para la transmisión del discurso instruccional. La clasificación y la enmarcación constituyen los principios generativos que regulan la forma y la ubicación específica de los sujetos en el discurso instruccional mediante las prácticas instruccionales. Así, cuando existe una clasificación fuerte cada discurso articula su "propia cultura" su "propia moral" y su "propia identidad". La clasificación fuerte entre discursos específicos crea relaciones de poder entre ellos. Estas relaciones de poder generan una organización jerárquica de discursos específicos y, por lo tanto, subordinaciones entre unos y otros. Así, por ejemplo, los discursos y prácticas vocacionales como un conjunto rígidamente clasificado de discursos/prácticas generan su propia "cultura técnica", su propia "moralidad técnica", su propia "identidad" y sus propios "espacios" 265.

La clasificación- fuerte entre discursos afecta la organización vertical y horizontal de los maestros, e indirectamente afecta el contexto escolar. La jerarquía vertical de los

\footnotetext{
${ }^{264}$ Posiblemente existe una homología entre la ubicación de discursos/prácticas expresivas jerárquicamente organizadas en la escuela y las disposiciones creadas en la ubicación dentro de tales discursos/practicas.

En otras palabras, en la selección y organización jerárquica de los discursos/practicas subyace un principio de clasificación de las disposiciones estéticas que actúa selectivamente sobre la ubicación de los sujetos en "el gusto" (disposiciones cultivadas/disposiciones no cultivadas, apelando a Bourdieu) (véase Bourdieu, 1979).

${ }^{265}$ Interesantes interrogantes han sido planteados por Grignon C (1971), en relación con este problema, en sus análisis de la cultura técnica en los niveles inferiores de la educación vocacional en Francia.
} 
maestros reproduce la organización jerárquica de los discursos (materias, cursos, unidades) y las posiciones asignadas a los maestros en un discurso (Grignon, 1971). La clasificación fuerte de los discursos instruccionales también produce diferentes redes especializadas de comunicación entre aquellos que controlan su administración (jefes de departamento, p.e.) y aquellos que lo reproducen, generándose de esta manera las relaciones de poder. Las relaciones horizontales creadas por la clasificación fuerte de los discursos separa o aisla a los maestros en discursos/espacios específicos y regula la forma que sus interacciones toman. Sin embargo, los jefes de departamento o equivalentes a pesar de estar clasificados por discursos tienen formas de interacción diferentes basadas en su "poder discursivo". En esta forma, las relaciones horizontales contribuyen a la reproducción de las relaciones verticales.

Cuando existe una clasificación débil (flexible) los límites que asignan a los discursos sus posiciones específicas se debilitan. La demarcación rígida entre diferentes zonas de discurso pueden ser desafiadas y la perturbación en la clasificación conduce a una perturbación en las estructuras existentes de la autoridad, en las identidades educativas específicas y en los conceptos de propiedad (Bernstein, 1977: 100-107). En este caso, la redistribución del conocimiento puede implicar cambios o transformaciones en las posiciones y disposiciones de los transmisores y los adquirientes.

El principio de enmarcación se refiere, básicamente, a la regulación de las prácticas instruccionales de transmisión/adquisición del discurso instruccional. En un sentido general, las prácticas instruccionales puede describirse como la ubicación y socialización de los adquirientes en significados específicos, seleccionados, abstraídos y reenfocados mediante el proceso de recontextualización ligado a la reproducción de competencias específicas. Los significados organizados en la forma de discursos instruccionales produce las bases para la realización diferencial de las competencias - y de sus rasgos internos y relacionantes- de los adquirientes.

En una forma más específica, las prácticas instruccionales pueden considerarse como prácticas especializadas de interacción que regulan la transmisión/adquisición/evaluación del discurso instruccional. Estas prácticas instruccionales presuponen la existencia de categorías especializadas de transmisores y adquirientes y de prácticas especializadas de interacción entre ellos.

Las relaciones sociales de transmisión/adquisición/evaluación del discurso instruccional específico, están reguladas por las siguientes reglas básicas:

1. Reglas de relación social que determinan la forma de las relaciones de poder entre transmisores y adquirientes.

2. Reglas discursivas que regulan la selección, secuencia, ritmo y criterios de la transmisión.

Estas reglas definen las formas de control que los transmisores y los adquirientes pueden tener sobre la transmisión/adquisición del discurso instruccional.

Desde la perspectiva analítica de Bernstein es posible describir las variaciones en las relaciones sociales de instrucción en términos de las variaciones en las reglas jerárquicas y de las variaciones en las reglas discursivas. Detrás de las variaciones en estas reglas están las variaciones en el principio de clasificación y en sus ideologías subyacentes intrínsecas. Así, por ejemplo, las reglas de secuencia explícitas pueden producir una 
clasificación temporal fuerte del proceso de la transmisión del discurso en el sentido de que su representación concreta está separada de su representación abstracta en el tiempo. Es decir, los rasgos superficiales del discurso están disponibles para los primeros estadios de la transmisión mientras que la gramática subyacente o los principios organizativos están disponibles para períodos posteriores. Si tal secuencia explícita se combina con un ritmo fuerte, entonces, aquellos niños que en virtud de sus orígenes de clase o de familia fallan en el dominio de las reglas de secuencia en los primeros años de su educación probablemente fallan en el dominio de las reglas de secuencia en los primeros años de educación probablemente fallen en el dominio de las reglas de secuencia más tarde, en su carrera educativa y, en consecuencia, no tengan acceso a la gramática subyacente o a las reglas generales que organizan el discurso. Aquí, el discurso instruccional reproduce la separación entre las prácticas manuales y las prácticas intelectuales (orientaciones restringidas Vs. orientaciones elaboradas) en su transmisión, y ubica al mismo tiempo las posiciones prospectivas de los alumnos en el campo de producción. Además, el ritmo fuerte requiere que todos los adquirientes efectúen trabajos escolares fuera de la escuela, en la casa. El fuerte ritmo del aprendizaje que imprime la escuela requiere dos sitios de adquisición, uno en la escuela y otro en el hogar. El origen socio-cultural de la familia actúa selectivamente sobre las posibilidades que ésta tiene como segundo sitio de adquisición.

Los efectos sociales del ritmo fuerte y de las reglas de secuencia explícitas son entre otros, el fracaso escolar, la clasificación por "habilidades", el bajo nivel de retención escolar y la presencia de discursos "reparadores" (modalidades compensatorias o remediales) tanto en la escuela como en instituciones especializadas para aquellos alumnos que no responden a los requerimientos de las reglas de transmisión.

Las reglas de secuencia explícitas, el ritmo fuerte y los criterios explícitos son generados por los valores internos fuertes de la enmarcación (+) pero tales valores de la enmarcación incluyen prácticas regulativas específicas.

Las relaciones entre las reglas de relación social y las reglas discursivas pueden esquematizarse en la siguiente figura:

\section{BASES REGULATIVAS PARA LAS PRACTICAS INSTRUCCIONALES}

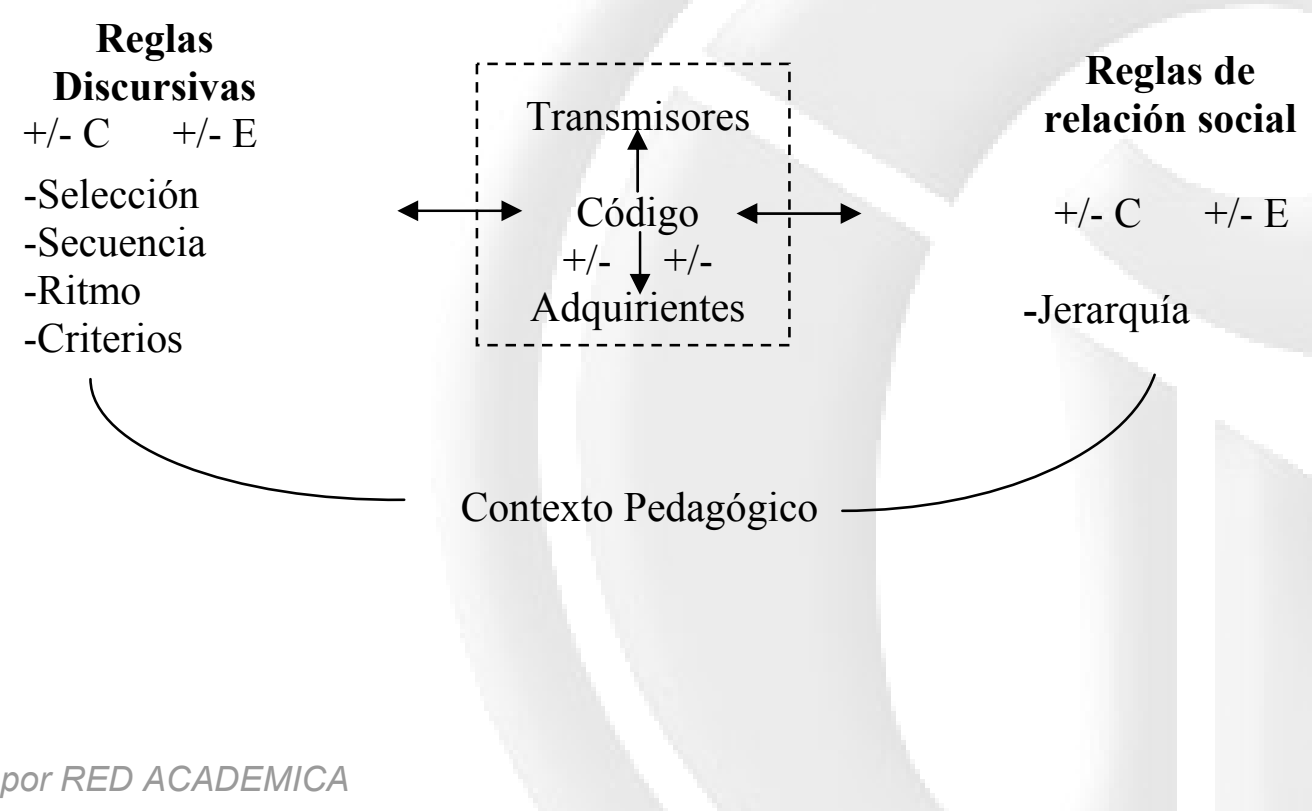




\section{Variaciones en el código de las prácticas instruccionales}

Podemos describir los códigos específicos que regulan la ubicación de los sujetos en el discurso instruccional en términos de la orientación hacia los significados y en términos de las realizaciones (valores de $\mathrm{C}$ y de $\mathrm{E}$ ) producidas por prácticas de interacción especializadas que constituyen los contextos comunicativos, (Bernstein, 1981). Desde esta perspectiva, la modalidad de un código instruccional se da mediante los valores de la clasificación y de la enmarcación. Así, las variaciones en el principio de clasificación de las categorías específicas (transmisores, adquirientes, discursos) varían desde $+C$ hasta - C. Las variaciones en el principio de enmarcación varían desde $+E$ hasta $-E$. Es importante agregar, siguiendo a Bernstein (1981) que "la fuerza de la clasificación y la fuerza de la enmarcación pueden variar independientemente la una de la otra" y que "cualquier conjunto de valores para la clasificación y para la enmarcación constituyen la modalidad del código".

De acuerdo con ésto, la gramática básica para la transmisión del discurso instruccional específico se proporciona a través de las relaciones entre la clasificación y la enmarcación y puede expresarse así:

$\begin{array}{ll}+C & +E \\ +C & -E \\ -C & +E \\ -C & -E\end{array}$

Esta gramática básica puede usarse para expresar las variaciones en la modalidad de transmisión/adquisición. Así, la clasificación puede referirse al acto de aprendizaje en el espacio del salón de clase, ya sea dicho acto aislado $(++C)$, interactivo dentro de grupos homogéneos $(+\mathrm{C})$, o dentro de grupos de diferente edad, género o habilidad $(-\mathrm{C})$. Esta clasificación básicamente concierne a los adquirientes. También nos podemos referir a la clasificación de los transmisores y de los discursos. Dentro de estos últimos, la clasificación puede extenderse a unidades menores, los pedagogemas, o mínimas unidades de significado susceptibles de ser evaluadas.

Podemos, entonces, encontrar las siguientes variaciones en la clasificación de los discursos, transmisores y adquirientes:

$\begin{array}{ccc}\begin{array}{c}\text { Discursos } \\ \text { (dentro de } \\ \text { y entre) }\end{array} & \begin{array}{c}\text { Acto de adquisición } \\ \text { (Adquirientes) }\end{array} & \text { Transmisores } \\ & & \text { aislado }+\mathrm{C} \\ -\mathrm{C} & +\mathrm{C} \text { grupos homogéneos } & -\mathrm{C} \\ & -\mathrm{C} \text { grupos heterogéneos } & \end{array}$

Las variaciones en la jerarquía, selección, secuencia, ritmo y criterios están reguladas, como dijimos, por la enmarcación. De acuerdo con esto, las variaciones pueden ser:

$\begin{array}{cll}\text { Reglas de relación social } & \text { Jerarquía } & +E-E \\ \text { Reglas discursivas } & \text { Selección } & +E-E \\ & \text { Secuencia } & +E-E \\ & \text { Ritmo } & +E-E \\ & \text { Criterios } & +E-E\end{array}$

En la realización de estas reglas puede ocurrir un conjunto de variaciones. Así, es posible encontrar una enmarcación fuerte sobre la selección y una enmarcación 
relativamente flexible sobre las reglas de secuencia y ritmo. Igualmente es posible considerar que la enmarcación fuerte sobre los criterios, en general, determina una enmarcación fuerte sobre la selección.

Es importante anotar que no todas las realizaciones son posibles. Algunas de éstas son contradictorias e inaceptables. Así, por ejemplo, una realización del tipo:

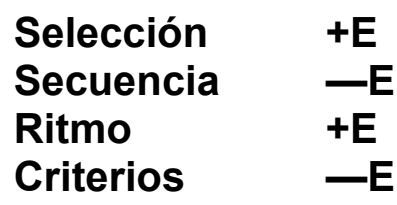

no es lógicamente posible en el salón de clase.

Existe una relación de articulación entre la gramática intrínseca del código y sus realizaciones ideológicas. Desde este punto de vista es posible considerar que el amplio rango de variaciones en la realización del código instruccional se relaciona con las ideologías instruccionales y sus teorías. Las teorías intrínsecas a una modalidad del código pueden variar en la forma como apuntan al enfoque y a los principios, medios contextos y posibilidades tanto del campo de producción como del campo de control simbólico.

En este sentido, el problema consiste en determinar qué rasgos y valores del código son afectados: así, por ejemplo, podemos distinguir variaciones en los valores de la clasificación de los agentes (transmisores/adquirientes), o podemos distinguir variaciones en los valores de la clasificación de los discursos como cuando oponemos el discurso académico de la educación formal al discurso no académico de la educación no-formal, o a los discursos/prácticas para los niños/alumnos de los sectores marginados.

La gramática básica del código instruccional puede representarse mediante la siguiente fórmula que expresa las relaciones fundamentales entre $C$ y $E$, y sus valores internos y externos:

\section{\pm Cta \pm Eie}

Podemos distinguir, igualmente, variaciones en la micro-relaciones de los valores externos de la enmarcación de las variaciones en sus macro-relaciones. Las microrelaciones de los valores externos de la enmarcación conciernen a la regulación de las relaciones entre la escuela y la familia/comunidad; las micro-relaciones también proporcionan una dimensión de la fuerza de los límites entre el conocimiento escolar y el conocimiento no-escolar. Las macro-relaciones de los valores externos de la enmarcación se refieren a la regulación de las relaciones entre la educación y la producción.

\section{Valores externos de la enmarcación: micro-relaciones}

Aquí desarrollaremos este análisis haciendo más explícitas las implicaciones de las reglas intrínsecas al código que regula la ubicación de los sujetos dentro de un discurso instruccional específico.

Cuando el código pedagógico tiene los valores generales de + C y + E entonces, en los sistemas educativos modernos es probable que exista un ritmo fuerte y explícito en el aprendizaje. Debemos tener en cuenta que el ritmo no es intrínseco a los discursos 
especializados que la escuela debe transmitir y, por lo tanto, no puede derivarse de la lógica de estos discursos. El ritmo explícito o implícito constituye una derivación de una teoría de la instrucción cuya selección es, en última instancia, un rasgo del discurso regulativo. El ritmo siempre tiene consecuencias regulativas en la definición del texto pedagógico que debe escribirse, hablarse, hacerse visual y evaluarse. En la medida en que el código presupone un ritmo fuerte se crea la necesidad de dos espacios de adquisición, la escuela y la familia, puesto que lo que debe aprenderse no puede ser ni enseñado ni adquirido totalmente en el tiempo escolar. Esto significa que los alumnos deben realizar tareas escolares fuera de la escuela de manera creciente a medida que aumentan en su escolaridad.

Podemos ver, entonces, que el ritmo fuerte presupone un control del alumno en el tiempo y en espacio extraescolar. De esta manera se extiende o ensancha la vigilancia pedagógica sobre los niños/alumnos y se incrementa la especialización de sus prácticas. En esta forma, el ritmo fuerte subordina a los alumnos al discurso pedagógico y, por lo tanto, a las prácticas oficiales reproductivas ya sea en la escuela, ya sea fuera de ella.

Cuando la familia no puede construir mínimamente un espacio pedagógico oficial o una práctica pedagógica oficial en su hogar es muy probable que el alumno no pueda adquirir las reglas de secuencia del código pedagógico dominante y que, por lo tanto, se vaya retrasando. Además, puede que exista una oposición entre las demandas del espacio pedagógico oficial y los rasgos de lo que hemos denominado espacio pedagógico local dependiendo de la ubicación social, regional, religiosa o racial de la familia. El espacio pedagógico local y sus prácticas constituyen el contexto donde el aprendiz se socializa en prácticas instruccionales y regulativas y donde reproduce actuaciones loca les legítimas sobre la base de competencias universales compartidas. Las prácticas pedagógicas locales presentes en la socialización de los niños son difusas y no están sometidas ni a un tiempo ni a un espacio especializados. En este sentido, pueden considerarse prácticas débilmente clasificadas.

A diferencia del espacio pedagógico local, el espacio pedagógico oficial es un espacio muy especializado y, además, fuertemente clasificado allí donde el código es $+C+E i^{e}$. En este caso es muy probable que el silencio sea requisito indispensable del espacio; así, el espacio instruccional demanda un fuerte aislamiento de los espacios ruidosos. Igualmente, es muy probable que se requiera una enmarcación fuerte de los valores externos lo cual significa que no debe haber comunicación entre el espacio pedagógico oficial y el espacio pedagógico local. Este requisito puede crear tensiones y conflictos, especialmente, cuando se inserta un espacio pedagógico oficial en la familia, inserción que no siempre es materialmente posible.

Si el espacio pedagógico oficial es una condición necesaria para la adquisición del código pedagógico oficial no es siempre una condición suficiente. La adquisición del código pedagógico oficial requiere de una práctica pedagógica oficial que regule la comunicación dentro de, y entre espacios. Aquí, es posible que se presente una nueva oposición o ausencia de relación entre la práctica pedagógica oficial y la práctica pedagógica local. Si la práctica pedagógica oficial está regulada por $+0+\mathrm{E}^{\mathrm{i}}$ e, entonces ella definirá el contexto social de aprendizaje como un contexto aislado, privatizado y competitivo, en oposición al contexto de la práctica pedagógica local, compartido y comunal. Los valores externos de la enmarcación serán, en este caso, fuertes, para excluir aquellas prácticas que no reproducen los principios organizativos de la escuela. 
El código pedagógico $+0+\mathrm{E}^{\mathrm{i}} \mathrm{e}$ necesariamente vincula a la familia como un segundo sitio crucial para el aprendizaje en una relación directa a la fuerza del ritmo si se desea que el código sea efectivamente adquirido. Los fundamentos de clase del ritmo generan la necesidad de una transmisión económica y de un principio de adquisición selectivo. El aprendizaje sólo puede ser efectivo allí donde la familia es colonizada por el discurso pedagógico oficial. La regla de ritmo, un rasgo aparente del discurso instruccional y a menudo justificada mediante sus teorías objetivas es portadora del discurso regulativo implícito tanto en la familia como en la escuela. Tenemos, entonces, que si el discurso pedagógico es un discurso incluido DI

$\mathrm{DR}$

DL

también es un discurso incluido en la familia que toma la forma de (Bernstein, 1981). DPO'

En el análisis de las micro-relaciones de los valores externos de la enmarcación hemos podido observar la penetración de la escuela en la familia a través del código que regula la posición de los sujetos en el discurso instruccional. Esto puede resumirse en el siguiente esquema: (Véase página siguiente).

\section{Valores externos de la enmarcación: macro-relaciones}

Hemos dicho que las macro-relaciones de los valores externos de la en-marcación se refieren a las relaciones entre la educación y la producción. Esto es, dichas macrorelaciones se refieren al grado en el cual la enmarcación en la educación puede fortalecerse con respecto al campo de producción, o puede debilitarse "con el propósito de acomodarse a las demandas o requerimientos de éste, en tal forma que se fortalezcan las relaciones sistémicas, —o de correspondencia- entre los productos de la educación y las demandas del trabajo" (Bernstein, 1981: 25).

Desde esta perspectiva, el fortalecimiento de los valores externos de la enmarcación $\left(+E^{e}\right)$ puede conducir a un debilitamiento de las relaciones sistémicas entre la educación y la producción, y un debilitamiento de los valores externos de la enmarcación $\left(-E^{e}\right)$ puede conducir a un fortalecimiento de las relaciones sistémicas, especialmente, con respecto a las modalidades dominantes en la educación ${ }^{266}$

Las macro-relaciones de los valores externos de la enmarcación, esto es, las relaciones sistémicas, pueden variar independientemente del principio fundamental de clasificación entre educación y producción.

Es importante agregar que las modalidades de control que surgen de los cambios en las macro-relaciones de los valores externos de la enmarcación pueden conducir a cambios en las micro-relaciones, esto es, en la enmarcación del qué y el cómo de la comunicación entre la escuela y la familia/comunidad.

\footnotetext{
${ }^{266}$ Es importante tener en cuenta que las relaciones sistémicas entre educación y producción se traducen en una motivación del niño hacia la educación, independientemente de la relevancia inmediata de dicha educación, o del nivel de actuación del niño. A su vez, dicha motivación se fundamenta en la ideología de la movilidad socia y se controla con la ideología de la meritocracia.
} 


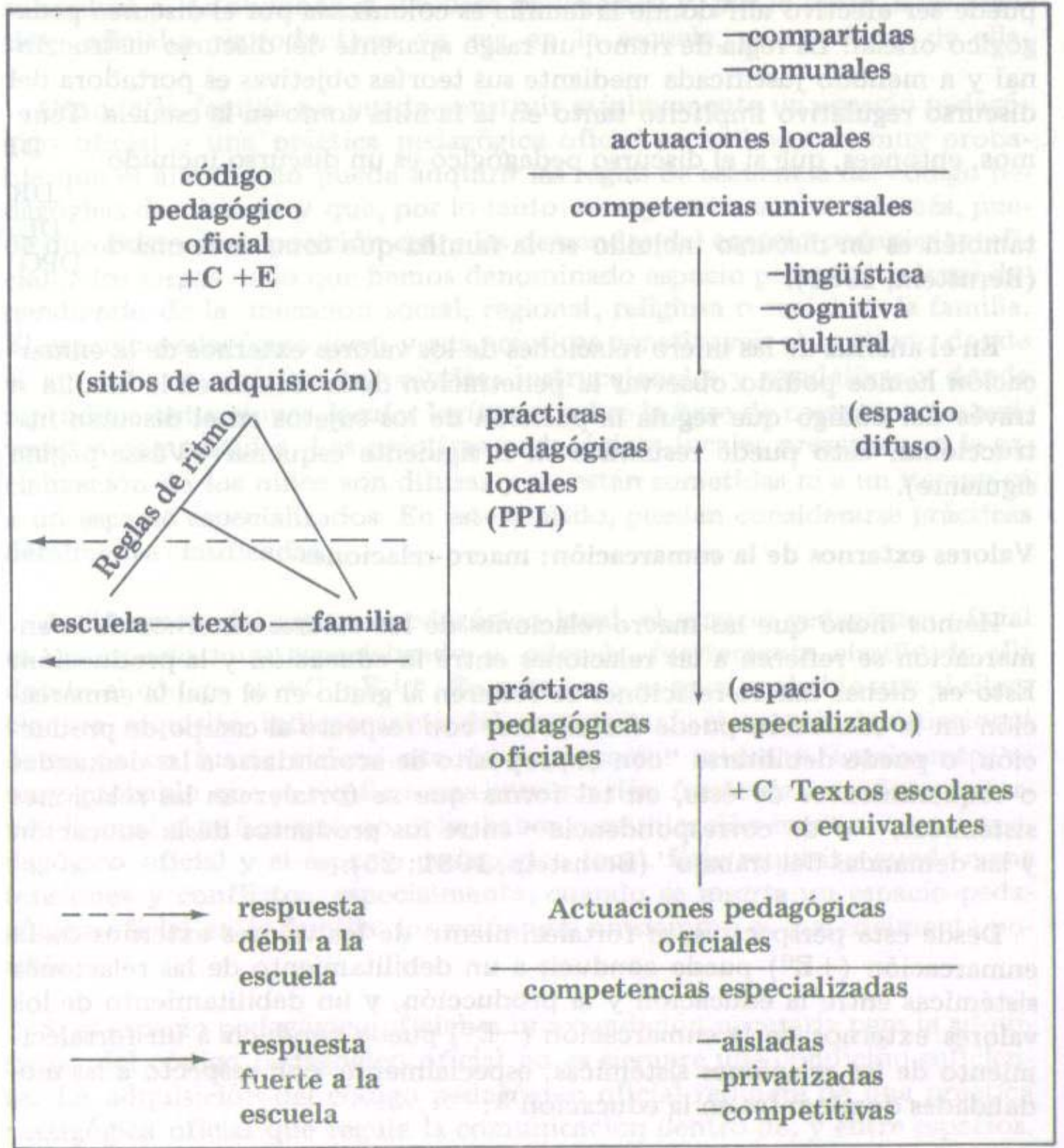

Las macro-relaciones de los valores externos de la enmarcación pueden afectar las variaciones del código instruccional tanto en sus modalidades dominantes (especialmente en los niveles más altos de la instrucción) como en sus modalidades dominadas. Estas últimas incorporan categorías, prácticas y contextos del campo de producción al campo de educación en la forma de discursos/prácticas vocacionales que proporcionan las habilidades y destrezas ocupacionales y sociales básicas. Este puede ser el caso del sistema de adiestramiento en instituciones corno el SENA o los INEM en Colombia.

\section{Conclusiones}

En este artículo hemos tratado de explicar cómo la ubicación del discurso instruccional y la ubicación dentro del discurso instruccional son creadas, legitimadas y reproducidas por la división social del trabajo y sus relaciones sociales intrínsecas. La ubicación de los discursos instruccionales en la escuela se basa en la división social del trabajo que establece la clasificación, ordenamiento y distribución de los discursos y que regula sus relaciones verticales y horizontales. La ubicación dentro de los discursos instruccionales 
presupone relaciones sociales específicas de transmisión! adquisición, esto es, presupone principios de control y sus respectivos valores ( $C$ y $E$ ).

La ubicación de un discurso instruccional específico constituye la matriz de transmisión y sus prácticas instruccionales intrínsecas. También presupone las competencias de los sujetos que acceden a dicho discurso. Es en este sentido que Bernstein define al discurso instruccional como el discurso que regula las reglas que constituyen la variedad legítima y los rasgos internos y relacionantes de competencias especializadas.

La ubicación del discurso instruccional en la escuela presupone relaciones de poder y reglas de recontextualización. De manera esencial, el poder puntúa la conciencia mediante los silencios producidos por los aislamientos de los discursos provocados por el principio de clasificación. Bernstein dice que "los intervalos, rupturas, separaciones que establecen categorías de semejanza y diferencia, de igual y de no-igual, son puntuaciones escritas por las relaciones de poder que establecen como el orden de las cosas a sujetos distintos con voces distintas" (Bernstein, 1981:13). Los aislamientos sirven para crear un orden externo supuestamente inviolable, natural e inevitable, al tiempo que intentan crear un orden interno a los transmisores y adquirientes mediante la supresión de las contradicciones inherentes al mismo principio de clasificación.

La ubicación dentro de un discurso instruccional específico presupone principios de control. Los principios de control seleccionan realizaciones (valores de la clasificación y de la enmarcación) que permiten la reproducción de la distribución del poder ligada a la distribución de los discursos instruccionales en la escuela. El control regula el principio de enmarcación y de esta manera, la gramática interna de la transmisión/adquisición de un discurso instruccional específico. Aquí, es importante reconocer que la adquisición de una determinada modalidad del código envuelta en el acceso a un determinado discurso instruccional es una fuente potencial de cambio de tal código a través de las tensiones que se crean entre el orden que dicho discurso presupone y las resistencias de los sujetos a la inclusión en dicho orden.

Si necesitarnos saber si el cambio de una modalidad de clasificación del discurso instruccional es un cambio en la distribución del poder, entonces, necesitarnos dar respuesta a estos interrogantes.

a. ¿A qué intereses responde el cambio en la clasificación?

b. ¿Qué otras clasificaciones retienen sus valores anteriores?

Cualquier modalidad de reproducción de competencias especializadas necesariamente presupone una lógica del poder y del control, tácitamente incluida en la adquisición de, o acceso a, los principios de un discurso instruccional específico.

\section{REFERENCIAS}

ADSHEAD, J. (1981) The study of dance, London: Dance Books Ltda.

BERNSTEIN, B. (1966) "Sources of Consensus and Disaffectlon ini Education" en, Bernstem, B. (1977) Class Codes and Control Vol. 3. Towards a theory of Educational Transmissions.

London: RKP. 
-. (1977) Class, Codes and Control, Vol. 3 Towards a Theory of Educational Transmissions,

London: R.K.P.

- (1981) "Codes, Modalities and the Process of Cultural Reproduction: A Model", Anglo American Studies Vol. 1, No. 1. (Traducción disponible en español).

-. Seminarios.

BERNSTEIN, B. y DIAZ M. (1985) "Hacia una teoría del Discurso Pedagógico" Revista Colombiana de Educación No. 15.

BOURDIEU, P. (1968) "Intellectual field and creative project" en Young, M.F.D. (1981) Knowledge and Control London: MacMillan.

BOURDIEU P. y PASSERON, J. (1977) Reproduction in Education, Society and Culture, London: Sage Publications Inc.

BOURDIEU, P. (1979) La Distinction, Critique Sociale du Jugement. París: Mlnuit.

DURKHEIM, E. (1977) The evolution of educational thought: Lectures on the formation and development of secondary education in France. London: R.K.P. (originalmente publicado en 1938).

GOODSON, I (1981) "Becoming and academic subject: Patterns of explanation and evolution"

British Journal of Sociology of Education V. 2 No. 2;

GOULDNER, A. W. (1976) The dialectic of ideology and technology: The origins, grammar and future of ideology, London: MacMillan Press Ltd.

GRIGNON C. (1971) L'ordre des choces: les fon ctlons sociales de l'enseignement technique. París: Minuit.

GREEN, M. (1971) "Curriculusn and Consciousness", The Record F 3 (2).

HIRST, P.H. (1965) "Liberal Education and the Nature of knowledge" en Archambault, R.D. (1965) Philosophical analysis and Education. London: R.K.P.

(1969) "The logic of the curriculum" Journial of curriculum studies 1.

HIRST, P.H. y PETERS, R.S. (1970) The logic of the curriculum, London: R.K.P.

PARSONS, T. (1951) The Social System, London: R.K.P.

PARSONS, T. BALES, F. et al. (1955) Family: Socialization and interaction Process. Glencoe, illinois: The Free Press.

PARSONS, T. (1959) "The School class as asocial system: some of its functions in American Society" H.E.R. 29. 
PETERS, R.S. (1965) "Education as Inltlation" en Archambault, R.D. (1965) Philosophilcal Analys/s and Education. London: R.K.P.

POULANTZAS, N. (1976) Las clases sociales en el capitalismo actual México: Siglo XXI Ed.

SKILBECK, M. (1980) Core Curriculum for Australian Schools. Camberra: Curriculum Development Centre.

WRIGHT, E.O. (1978) Class, Crisis and the State. London: NLB.

YOUNG, M.F.D. (1975) "Curriculum change: limits and possibllities" Educational studies, (12).

VULLIAMY, G. (1976) "What counts as school music? en Whltty, G. y Young M. (eds.) 1976 Explorations in the Politics of school knowledge, Nafferton: Nafferton books. 